
\title{
$\begin{array}{ll}\text { Research Square } & \text { They should not be connsidered conchlusive, used to i inorrm clinical practice, } \\ \text { or referenced by the media as validated information. }\end{array}$
}

\section{Insights from shotgun metagenomics into bacterial species and metabolic pathways associated with NAFLD in obese youth}

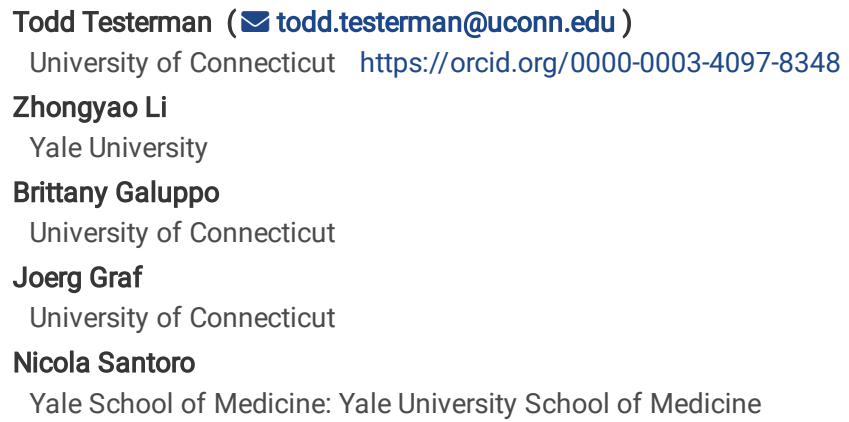

Research Article

Keywords: microbiome, Bacteroides, Fusicatenibacter, branched chain amino acid, aromatic amino acid, fermentation, Romboutsia, short chain fatty acid, Odoribacteriaceae, Eubacterium

Posted Date: November 15th, 2021

DOI: https://doi.org/10.21203/rs.3.rs-1065165/v1

License: (c) (1) This work is licensed under a Creative Commons Attribution 4.0 International License. Read Full License

Version of Record: A version of this preprint was published at Hepatology Communications on March 28th, 2022. See the published version at https://doi.org/10.1002/hep4.1944. 


\section{Abstract \\ Background and Aims}

NAFLD is the most common form of liver disease and is often the precursor for more serious liver conditions such as NASH and cirrhosis. The gut microbiome has been implicated in the development of NAFLD, but studies have often compared healthy individuals to those with the disease. In this study, we aimed to examine the taxonomic and functional differences between the microbiomes of obese youth with and without NAFLD.

\section{Approach and Results}

Shotgun metagenome sequencing was used to profile the microbial communities of 36 subjects, half of which were diagnosed with NAFLD. Beta diversity analysis showed community-wide differences between the cohorts $(\mathrm{p}=0.002)$. Specific taxonomic differences included the species Fusicatenibacter saccharivorans ( $\mathrm{p}=0.042)$, Romboutsia ilealis $(\mathrm{p}=0.046)$, and Actinomyces sp. ICM47 ( $p=0.0009)$ elevated in the NAFLD-positive group and Bacteroides thetaiotamicron $(p=0.0002)$ elevated in the NAFLD-negative group. At the phylum level, Bacteroidetes $(p<0.0001)$ was elevated in the NAFLD-negative group. Functionally, branched chain amino acid $(p=0.01343)$ and aromatic amino acid $(p=0.01343)$ synthesis pathways were elevated in the NAFLD-positive group along with numerous energy utilization pathways including pyruvate fermentation to acetate $(p=0.01318)$.

\section{Conclusions}

The link between obesity and NAFLD development in younger individuals has rarely been probed, with most studies focusing on adults and comparing healthy individuals with obese, NAFLD-positive individuals. This study supports the idea that the NAFLD phenotype displays a differentiated microbial and functional signature from the obesity phenotype.

\section{Introduction}

Nonalcoholic fatty liver disease (NAFLD) is the most common hepatic complication of obesity in children and adolescents. It is estimated that about $34 \%$ of youth with obesity is affected by NAFLD(1-3). Moreover, data based on histological specimens have shown that inflammation and fibrosis may occur early in life in patients with NAFLD(4), making them susceptible to develop liver failure during the second decade of life(5). Although NAFLD is a common complication of obesity in youth, the reason why some patients are susceptible to the disease while other patients with obesity will never develop it, is still unclear. Nutritional and genetic factors certainly play a key role in conveying susceptibility to NAFLD(6-8), but other factors may drive individuals to develop this condition. In particular, it has been suggested that the composition of the gut microbiota may play a pivotal role in this regard(9,10).

Previously, some studies have shown associations between intrahepatic liver content and gut microbiota(10). Seminal twin studies in adults with NAFLD have demonstrated that within the spectrum of NAFLD, a metagenomic signature can differentiate not only between subjects with differing degrees of fibrosis(11), but also between subjects with and without cirrhosis(12).

This and other findings led us, in a previous study, to assess whether the gut microbiota could be different between youth with and without NAFLD(13). We showed that youth with NAFLD have a different metagenomic profile than those without NAFLD(13). Our initial analysis was limited by sequencing the V4 region of 16S rRNA gene, which typically allows for family- and genus-level identification and no direct assessment of the physiological potential of the microbiome.

Shotgun metagenome studies, wherein the entire fecal DNA content is sequenced, that have focused on the NAFLD phenotype show a clear distinction between the microbial communities present, though the identified contributing taxonomic groups have varied widely. Studies have found bacterial phyla including Proteobacteria, Firmicutes, and Bacteroidetes, along with specific bacterial genera including Escherichia, Veillonella, Faecalibacterium, Eubacterium, Bacteroides, and others to be associated with NAFL disease progression $(11,14,15)$. Shotgun data, providing functional potentials for the native microbial community, has associated metabolic pathways including branched chain amino acid (BCAA) biosynthesis, aromatic amino acid (AAA) biosynthesis, fatty acid biosynthesis, and nucleotide biosynthesis with NAFLD status $(14,15)$.

In the present study, we sought to follow-up on our previous findings by shotgun-sequencing and analyzing the metagenome of obese patients with and without NAFLD. Within this group of obese subjects, we observed community-wide differences in metagenome composition with specific species implicated in driving these differences. We also identified metabolic pathways enriched in obese, NAFLD-positive and NAFLD-negative groups and the species contributing to these enrichments.

\section{Results}

\section{Population Characteristics Stratified by NAFLD Status}

Baseline clinical and demographic characteristics were investigated by NAFLD status in Table 1 for 36 subjects (18 NAFLD-positive and 18 NAFLD-negative). Chi-square tests and two-sample t-tests were used for categorical variables and continuous variables, respectively, to test the differences. A majority of the baseline characteristics were not significantly different between the subjects with NAFLD and subjects without NAFLD. Compared to the NAFLD negative group, subjects with NAFLD have higher BMI, BMI z-score, fasting insulin, ALT, visceral fat and hepatic fat fraction but lower WBISI. These all attained 
statistical significance $(\mathrm{p}<0.05)$. Although not statistically significant, subjects with NAFLD had elevated fasting blood glucose, body fat percentage, total cholesterol, triglycerides, and AST.

\section{Microbial Community Profile of 36 Obese Youth}

36 shotgun metagenomes consisting of 18 NAFLD-positive and 18 NAFLD-negative subjects were analyzed. Figure 1 compares alpha and beta diversity between these groups as well as profiling the microbial communities at the species and phylum levels. Shannon-Weiner diversity index values were calculated for all samples and was shown to be slightly elevated in the NAFLD-positive group (Fig. 1A). However, this difference was not shown to be statistically significant $(p=0.14$, t-test). Bray-Curtis dissimilarity values were calculated and plotted using an NMDS ordination to compare overall microbial community structure at the species-level (Fig. 1B). PERMANOVA analysis revealed that the samples of the NAFLD-positive group had significantly different clustering from the NAFLD-negative samples ( $\mathrm{p}$.adj $=0.001, \mathrm{R}^{2}=0.1006$, PERMANOVA). There was also a significant difference in dispersion ( $\mathrm{p}$.adj $=0.023, \beta$-dispersion). These results indicate that at the species level the difference between the centroids of these groups is significant, but this may be due to the tested factor (disease), variability within each group, or a combination of these two factors(16). Agglomerating at higher taxonomic levels (genus thru phylum) led to reduced and no longer significant dispersion differences while maintaining the significant clustering differences as measured by PERMANOVA testing (Supplemental Figure 1).

At the phylum level (Figure 1C, Supplemental Figure 2A), the average relative abundance of Firmicutes (mean relative abundance, NAFLD-positive vs. NAFLDnegative: $72.1 \%$ vs. $56.1 \%$ ) and Bacteroidetes (6.7\% vs. $33.2 \%$ ) differed greatly between non-diseased and diseased groups. Actinobacteria (18.8\% vs. $7.9 \%)$ was the third most prevalent phylum for both groups. Verrucomicrobia ( $0.9 \%$ vs. $1.9 \%)$ and Proteobacteria $(0.3 \%$ vs. $0.5 \%)$ were present as minor bacterial phyla along with the archaeal phylum Euryarcheota ( $0.8 \%$ vs. $0.4 \%)$ as well as viruses $(0.2 \%$ vs. $0.1 \%)$. At the species level (Figure $1 \mathrm{D}$, Supplemental Figure 2B), the Firmicutes Faecalibacterium prausnitzii (9.4\% vs. 12.7\%), Eubacterium rectale (8.6\% vs. $4.6 \%)$, Fusicatenibacter saccharivorans (6.0\% vs. $1.6 \%$ ), Ruminococcus bromii (3.9\% vs. 3.3\%), Anaerostipes hadrus (4.1\% vs. 3.0\%), Eubacterium sp. CAG 180 (2.4\% vs. $2.7 \%$ ), and Dorea longicatena (3.2\% vs. $1.7 \%$ ); the Bacteroidetes Prevotella copri (1.6\% vs. $10.0 \%)$, Bacteroides vulgatus (1.4\% vs. $7.5 \%)$; and the Actinobacteria Bifidobacterium adolescentis ( $8.5 \%$ vs. $2.3 \%$ ), represented the 10 most abundant species. The next 25 most abundant species belong to the genera Eubacterium, Ruminococcus, Colinsella, Roseburia, Bifidobacterium, Bacteroides, Lactobacillus, Allistepes, Akkermansia, Dorea, Blautia, Coprococcus, and Parabacteroides.

\section{Taxonomic Differences between NAFLD-negative and NAFLsD-positive Groups}

The differences between the microbial communities of NAFLD-negative and NAFLD-positive individuals were first compared using principal coordinate analysis (PCA) biplots. PCA plotting presents a compositional approach where results are generally more reproducible and variance in the data is directly accounted for within the plot (as opposed to requiring separate statistical analysis as in PCoA plotting)(17). Figure 2 shows the species and phylum-level PCA plots with arrows representing the contribution of individual taxa to each principal coordinate (the length of the arrow represents the strength of the effect). Beneath each PCA biplot is an iris plot showing the taxonomic composition for each sample corresponding to the location on the PCA plot above. The percent of the variation explained in the PCA plot increased from the species level (PC1 12.2\%, PC2 10.6\%) to the phylum level (PC1 57.1\%, PC2 23.0\%).

Species with a large effect size in the quadrant containing most of the non-NAFLD samples include Alistepes putredinis, Odoribacter splanchnicus, Barnesiella intestinihominis, Parabacteroides merdae, Bacteroides thetaiotamicron, and Bacteroides fragilis (Figure 2A, C). None of the top 10 species shown were dramatically enriched in the NAFLD-positive individuals. At the phylum level, Bacteroidetes is enriched in the area of the samples from the non-diseased group while Actinobacteria and viruses are enriched in the area of the samples from the diseased group (Figure 2B, D). Proteobacteria and Verrucomicrobia are enriched in a subset of both non-diseased and diseased individuals while Firmicutes does not have a clear correlation with either group.

The biplots suggested that certain taxa may correlate with the disease state of the subjects and differential abundance testing was then used to probe this in a statistically meaningful way. ANCOM with bias correction (ANCOM-BC) was used to determine if any taxa differed significantly in the microbiomes from NAFLD-positive and NAFLD-negative subjects. All taxonomic levels from species to phylum were tested. The results for this are presented in Table 2. The phylum Bacteroidetes, class Bacteroidia, order Bacteroidales, family Bacteroidaceae, and genus Bacteroides were all significantly more abundant in the NAFLD-negative cohort. One species was shown to be enriched in the NAFLD-negative cohort ( $B$. thetaiotamicron). Three species were shown to be enriched in the NAFLD-positive group including Fusicatenibacter saccharivorans, Romboutsia ilealis, and Actinomyces sp. ICM47.

The differentially abundant species identified using ANCOM-BC were also plotted in Supplemental Figure 3 as relative abundances in box plots. Certain species including Bacteroides thetaiotaomicron and Fusicatenibacter saccharivorans make up a larger portion of the average species consortium (>0.1\%). Others, including Actinomyces sp. ICM47 and Romboutsia ilealis, amount to a smaller portion (< $0.1 \%)$ of the microbial community. The significant results from all other taxonomic levels are plotted in Supplemental Figures 4-7.

\section{Functional Differences between NAFLD-negative and NAFLD-positive Groups}

In addition to taxonomic differences, functional differences between the groups were also investigated. Following functional classification of reads, the abundances were compared, and the top 50 statistically significant results are reported in Table 3. 29 of these pathways were elevated in the diseased group and 21 were elevated in the non-diseased group. Amongst these, a large number of biosynthetic pathways were identified including multiple amino acid synthesis pathways. In the NAFLD-positive group, multiple lysine synthesis pathways were elevated along with methionine, isoleucine, ornithine, threonine, serine, tryptophan, arginine, aspartate, and glycine pathways. Superpathways for BCAA synthesis and AAA synthesis along with peptidoglycan synthesis were also elevated in the NAFLD-positive group. In the NAFLD-negative group, ornithine (a different pathway than in the NAFLD-positive group), glutamine, glutamate, and isoleucine (a different pathway) were identified as elevated. Degradation, utilization, and energy generation pathways were also identified. Pyruvate fermentation to acetate and lactate, methanogenesis from acetate, lactose and galactose degradation, glycerol degradation, stachyose degradation, 
guanosine degradation, glycolysis, and the pentose phosphate pathway were elevated in NAFLD-positive individuals. Two histidine degradation pathways as well as the urea and TCA cycles were elevated in the NAFLD-negative individuals. A listing of every significant result can be viewed in Supplemental Table 1.

Additionally, the specific contributions of individual species to a particular metabolic pathway were calculated. These contributions were then compared between groups and differentially abundant taxonomic contributions were identified. Bar plots are provided in Supplemental Figures 9-14 for a subset composed of pathways of interest. Eubacterium hallii and Fusicatenibacter saccharivorans were by far the two most commonly seen taxa contributing to elevated pathways in the NAFLD-positive group. Additionally, Blautia wexlerae, Blautia obeum, Streptococcus salivarius, Ruminococcus torques, Streptococcus parasanguinis, Coprococcus catus, Streptococcus thermophilus, Romboutsia ilealis, Roseburia sp. CAG 471, and Dorea formicigenerans were also elevated in certain pathways. For the NAFLD-negative group, Bacteroides vulgatus, Bacteroides thetaiotaomicron, Bacteroides ovatus, Bacteroides stercoris,

Parabacteroides distasonis, Bacteroides fragilis, Bacteroides xylanisolvens, Ruthenibacterium lactatiformans, and Parabacteroides merdae were all elevated in specific pathways. All significant results can be viewed in Supplemental Table 1.

\section{Discussion}

In this study, we used shotgun metagenomic sequencing to illuminate a number of taxonomic and functional differences between the microbial communities in fecal samples of NAFLD-positive and negative individuals. Previous studies sequenced relatively short variable regions of the $16 \mathrm{~S}$ rRNA gene, which limits the taxonomic resolution capacity and lacks important functional information(18). The analysis of the shotgun metagenome data at the species level revealed that the composition of the microbiomes differed significantly between the NAFLD-positive and NAFLD-negative cohorts (Fig. 1, p.adj $=0.002, R^{2}=$ 0.1006 , PERMANOVA). In addition, we tested for differences in variability between the two groups and found that at the lower taxonomic levels (species and genus), variability was significantly different between the two groups (Figure 1B and Supplementary Figure 1A). This was not the case from the family level up to the phylum level (Supplementary Figure 1B-E). The high degree of variability of the human microbiome, especially at the species and genus levels, has been well documented $(19,20)$. This may explain the dispersion visualized in Figure 1B particularly for the non-diseased group. The reduction in variance at higher taxonomic levels suggests that in the non-NAFLD subjects a larger number of genera and species vary between individuals than in subjects with NAFLD.

Given the large number of different taxa present in microbiomes and the variability between individuals, it can be difficult to parse apart whether particular variables that drive the difference between youth with and without NAFLD. The taxonomic contributors of this observed difference were analyzed by differential abundance testing and displayed using PCA biplots. The taxa arrows presented on the biplots represent the most significant contributors to each principal coordinate. Interestingly, there seems to be a number of species that correlate with the non-diseased group whereas the NAFLD-positive group lacks obvious ones. The ANCOM analysis further supports this observation as identified taxa were all shown to be elevated in the NAFLD-negative group. This points to the potential that a lack of certain microbial taxa may contribute to the pathogenesis of NAFLD and thus be due to a dysbiotic state as opposed to specific taxa being causative(21). Alternatively, there could be a large number of different taxa that contribute to NAFLD, but they were not observed consistently enough to yield significant results. The most commonly observed and significant taxonomic difference between these two groups was the relatively increased abundance of bacteria belonging to the phylum Bacteroidetes in non-diseased subjects. Both the PCA biplot and ANCOM differential abundance testing identified this group as strongly enriched in NAFLD-negative individuals. Interestingly, a commonly cited overabundance of Proteobacteria(22) in NAFLD-positive individuals was not observed in our dataset though a subset of both study groups possessed elevated Proteobacteria. A number of species were identified as being differentially abundant and one of these, B. thetaiotamicron, was also identified as a major driver in PCA (Fig. 2). B. thetaiotamicron has been shown to reduce diet-induced body weight gain and adiposity in mice and was shown to be in lower abundance in obese human subjects compared to healthy(23). O. splanchnicus, a species within the family Odoribacteriaceae which was shown to be elevated in the NAFLD-negative group, has been associated with reduced incidence of $\operatorname{NAFLD(24),~cystic~fibrosis(25),~and~inflammatory~bowel~disease(26)~in~previous~studies.~This~species~}$ was identified as a major driver in PCA and its bacterial family was identified via ANCOM analysis. Fusicatenibacter saccharivorans, the lone species within a relatively newly recognized genus(27), comprised a fairly large portion of the NAFLD-positive community. This species has been associated with a diet high in processed foods(28) and is capable of fermenting a large diversity of saccharides, producing SCFAs as a result(27). Actinomyces sp. ICM47 is a species primarily associated with the human oral microbiome(29). Its overabundance in NAFLD-positive individuals could be due in part to increased saliva entering the GI tract whether through more frequent eating or an increased salivary response (though this is only speculation). It is important to note that detecting DNA from specific bacterial species does not establish their living presence within a certain niche. Oral microbial DNA found in fecal samples is to be expected but does not necessarily indicate that a species is a gut resident.

Functionally, an even more expansive list of features was shown to be differentially enriched between the two groups. BCAA synthetical (Supplementary Figure 9) and AAA synthetical (Supplementary Figure 10) genes were found to be elevated in the group with NAFLD and previous studies reported BCAA and AAA to be associated with $\operatorname{NAFLD}(30,31)$. Interestingly, isoleucine (a BCAA) pathways were shown to be elevated in both groups. Isoleucine pathway I (Supplementary Figure 11) and pathway III (Supplementary Figure 12) were elevated in NAFLD-positive individuals while pathway IV (Supplementary Figure 13) was elevated in NAFLD-negative individuals. Pathways 1 and 3 utilize 2-oxobutanoate and glutamate, respectively, while pathway 4 utilizes propanoate. Amongst these three pathways mentioned, contributions by Eubacterium, Blautia, and Streptococcus spp. were noted as overabundant in NAFLD-positive individuals while Bacteroides and Parabacteroides spp. were noted for NAFLD-negative individuals. While the detection of genes in the genomes does not imply that these genes are expressed, it is possible that these pathways are active and lead to an increase in free isoleucine associated with NAFLD.

The fermentation of pyruvate to lactate and acetate is another interesting pathway elevated in the NAFLD-positive group (Supplementary Figure 14). This difference is primarily driven by Fusicatenibacter saccharivorans and two Streptococcus species. Total short chain fatty acid (SCFA) content in the gut has been correlated with obesity status(21). With both of these groups being classified as obese, this may indicate that certain bacterial community members further exacerbate SCFA concentration, increasing chances for disease progression. The increase or decrease in specific SCFAs (acetate, propionate, butyrate) may also contribute to disease progression. Butyrate is noted as being the most potent anti-inflammatory SCFA whereas acetate is the least(32). The gene potential for increased acetate production by $F$. saccharivorans and a loss in butyrate production from decreased abundance of $F$. prausnitzii (the most

Page $4 / 15$ 
abundant species in these metagenomes and a known butyrate producer) (Supplemental Figure 2B), may play a role in elevated gut inflammation in the NAFLD-positive group(33).

We previously characterized a larger cohort that included the subjects presented in this study using V4 16S rRNA gene sequencing(13). The outcomes of that study included decreased alpha diversity in the NAFLD-positive group, no observed significant differences based on beta diversity, and a number of taxa identified as being differentially abundant between the groups. Of the identified taxa, the only overlap between studies was the overabundance of the phylum Bacteroidetes in NAFLD-negative individuals. These different study outcomes between a larger dataset and a subset of that data can have multiple contributing confounding factors. We compared the larger group and its subset used in this paper for all relevant metadata. Only race returned a significant result. Compared to the larger cohort, the cohort included in this study has less White and Hispanic individuals in the NAFLD-positive group. These differences may have contributed to the differing conclusions between the two studies as race is significant factor in microbial community composition(34,35). The techniques used in these studies (16S amplicon vs. shotgun sequencing) each provide their own benefits and drawbacks. Shotgun sequencing provides higher taxonomic resolution, is less prone to bias, can profile eukaryotic and viral constituents, and provides information on the functional potential of the native community $(36,37)$. However, it is significantly more costly due to requiring deeper sequencing depth to find rarer taxa and genes and to compensate for hostassociated reads(38). It can also be limited by the available genomes present in the database (e.g., NCBI). 16S rRNA gene sequencing is cost-effective and has less complex bioinformatic requirements(38). However, it is prone to primer biases which can skew the results or miss certain taxonomic groups and only provides taxonomic information on bacterial and some archaeal members $(36,37,39)$.

There are some important considerations to keep in mind with this dataset. One of these is the relatively small sample size $(n=36)$ examined in this study. This can lead to a potentially high false discovery rate (FDR) primarily due to outliers and random variability. To account for this, we did make FDR-related statistical adjustments and also used minimum prevalence values to exclude taxa or features only found in a small subset of samples. Another consideration, particularly for the functional data, is that shotgun metagenomic data is providing the functional potential of the community present as opposed to the expressed function. This is an important distinction as certain genes may be present but are not being actively transcribed while others may be overexpressed. Metatranscriptomics would be needed to acquire a true functional snapshot of the community, but this comes with its own set of caveats as the transcriptomes of fecal microbiome samples are not necessarily representative of the physiology in the colon. Lastly, parsing apart whether the observed microbial community differences between NAFLD-negative and NAFLD-positive groups is causative or correlative can be difficult. Dysbiosis is associated with the development of NAFLD and with obesity more broadly. The differences noted here may help further the understanding of the interplay between obesity, hepatic disease progression, and the microbiome.

This study profiled the microbial communities of a largely under-characterized age group within the population(34). The comparison examined was also unique as many studies include healthy individuals (non-obese and non-diseased) with NAFLD individuals. Our study included only individuals classified as youth with obesity with and without NAFLD as determined by MRI. This type of comparison is highly valuable as it illuminates potential causes as to why certain individuals develop disease while others do not. Another beneficial aspect of this study is the use of shotgun metagenomic sequencing to provide functional data along with taxonomic data. This reduces the level of extrapolation required to draw functional conclusions from $16 \mathrm{~S}$ rRNA gene datasets. Determining whether the microbiome is both correlative and causative or only correlative is difficult. Regardless, the connection between the microbial consortium residing within the human gut and NAFLD status is evident. Future studies that combine clinical, metabolomic, and microbiome data will be extremely important for prospective therapies for NAFLD and its more severe progressions, especially considering that the younger population is in the midst of an obesity epidemic.

\section{Methods Study cohort}

Thirty-eight youth with obesity (BMI $\geq 95$ th percentile) agreed to participate in this study. The participants underwent an oral glucose tolerance test (OGTT) and an abdominal fast-magnetic resonance imaging (MRI) to assess abdominal body fat partitioning and intrahepatic fat content as previously described(40-42). In addition, fasting blood samples to measure liver function tests and lipid profile were obtained. The studies were conducted at the Yale Center for Clinical Investigation at $0800 \mathrm{~h}$ after a 12-hour overnight fast. A stool sample from each subject was also collected. Written parental informed consent and written child assent were obtained from all participants. Yale University Human Investigation Committee approved the study.

\section{Shotgun Metagenome Sample Preparation and Sequencing}

DNA was extracted using the MoBio PowerMag Soil 96-well kit (MoBio Laboratories, Carlsbad, California) from $0.25 \mathrm{~g}$ of fecal sample. DNA extracts were quantified using the Quant-iT PicoGreen kit (Invitrogen, ThermoFisher Scientific). Metagenome libraries were produced using the TruSeq PCR-Free kit (IIlumina, San Diego, CA). Libraries were then diluted and pooled for loading onto an Illumina HiSeq 2500 . The libraries underwent $75 \times 75$ bp paired-end sequencing.

\section{Shotgun Metagenome Pre-Processing}

Raw paired end read data for 36 shotgun metagenomes were downloaded from Basespace (basespace.illumina.com) following demultiplexing. Libraries for two samples failed and were excluded from the study. The reads were then processed using KneadData (version 0.7.4,

https://github.com/biobakery/kneaddata) to remove adapter sequences, quality filter and quality trim reads, and remove contaminating host-associated reads. Default parameters were used throughout. The median read depth following QC was 46 million reads and the range was 69 million reads.

\section{Taxonomic Profiling and Comparison}

MetaPhIAn (version 3.0)(43) was used to taxonomically profile the microbial community to the species-level using the latest marker gene ChocoPhIAn database (release 2019.01). To investigate potential differences between the viromes of the two study groups, a virus profiling parameter was included as 
well. Individual sample taxonomic profiles were merged using the merge_metaphlan_tables.py utility script resulting in a single table with relative abundances provided for each taxon. To recover absolute counts for use in downstream analyses, the relative abundances resulting from the merge table script were multiplied by the total read count for each sample. Species-level counts were retrieved from this table and reformatted for import into R (version 4.1.0)(44).

An OTU table, taxonomy table, and metadata table were imported into RStudio (version 1.4.1717) and read into the package phyloseq (version 1.36.0)(45). Alpha and beta diversity calculations were performed using the packages phyloseq and microViz (version 0.7.7)(46). For ecological distance metrics, samples were rarefied to $8,893,658$ million reads (the read count of the lowest read count sample) before calculating distances and ordinating. For compositional biplots, a centered log-ratio transformation was performed as recommended before the generation of PCA biplots.

Differential abundance testing was performed using the ANCOM statistical framework $(47,48)$ implemented by the package ANCOM-BC $(49)$. Taxa were aggregated at the species, genus, family, order, class, and phylum levels using the microbiome package (version 1.14)(50) before performing the ANCOM test on each level. Taxa had to appear in a minimum of $25 \%$ of samples to be included in ANCOM-BC analyses.

\section{Functional Profiling and Comparison}

HUMAnN (version 3.0)(51) was used to functionally profile the microbial community. Paired end sequence files were first concatenated before running HUMAnN. The full ChocoPhIAn pangenome database (release 2019.01) was used for functional pathway abundance and coverage determination. The UniRef90 database (release 2021.03)(52,53) was used for gene family abundance determination. The output pathway and gene family abundance files for each sample were normalized to relative abundances and the resulting files were joined.

Enriched pathways and gene families were identified using the R package MaAsLiN2 (version 1.6.0)(54). Pathways and gene families achieving a corrected pvalue of 0.05 or less were classified as significantly enriched within one of the two patient groups. Identified pathways and gene families were then plotted using the bar plot utility script in HUMAnN.

\section{Abbreviations}

BCAA - branched chain amino acid

AAA - aromatic amino acid

PC - principal coordinate

ANCOM - analysis of compositions of microbiomes

$\mathrm{BMI}$ - body mass index

WBISI - whole-body insulin sensitivity index

AST - arginine transaminase

ALT - alanine transaminase

IGI - insulinogenic index

DI - disposition index

HDL Cholesterol - High-Density Lipoprotein Cholesterol

LDL Cholesterol - Low-Density Lipoprotein Cholesterol

IGT - Impaired Glucose Tolerance

NGT - Normal Glucose Tolerance

\section{Declarations}

\section{Funding}

This study was supported by the National Institute of Diabetes and Digestive and Kidney Disease (R01 DK113403-01A1), by the Yale Center for Clinical Investigation.

\section{Conflicts of Interest}

The authors have nothing to disclose.

Ethics Approval

Yale University Human Investigation committee approved the study. 
Consent to Participate and Consent for Publication

Written child assent and written parental permission were obtained from all participants involved in the study.

Availability of Data and Material

Data can be found in the NCBI SRA database under project ID PRJNA328258.

Code Availability

Code can be found at https://github.com/joerggraflab/Code-for-Testerman-Li-2021.

\section{Acknowledgements}

We are grateful to the clinical and research staff of the Hospital Research Unit (HRU), Church Street Research Unit (CSRU), and YCCI Core Lab at Yale School of Medicine.

\section{References}

1. Castillo-Leon E, Cioffi CE, Vos MB. Perspectives on youth-onset nonalcoholic fatty liver disease [Internet]. Endocrinology, Diabetes and Metabolism. 2020 [cited 2021 Jun 29];3. Available from: /pmc/articles/PMC7576279/

2. Marzuillo P, Giudice EM, Santoro N. Pediatric non-alcoholic fatty liver disease: New insights and future directions [Internet]. World Journal of Hepatology. 2014 [cited 2021 Jun 29];6:217-225. Available from: https://pubmed.ncbi.nlm.nih.gov/24799990/

3. E D, N S, S C. Metabolic syndrome in pediatrics: old concepts revised, new concepts discussed. Pediatric clinics of North America [Internet]. 2011 [cited 2021 Oct 24];58:1241-1255. Available from: https://pubmed.ncbi.nlm.nih.gov/21981958/

4. Patton HM, Yates K, Unalp-Arida A, Behling CA, Huang TTK, Rosenthal P, et al. Association between metabolic syndrome and liver histology among children with nonalcoholic fatty liver disease. American Journal of Gastroenterology [Internet]. 2010 [cited 2021 Jun 29];105:2093-2102. Available from: https://pubmed.ncbi.nlm.nih.gov/20372110/

5. Feldstein AE, Charatcharoenwitthaya P, Treeprasertsuk S, Benson JT, Enders FB, Angulo P. The natural history of non-alcoholic fatty liver disease in children: A follow-up study for up to 20 years. Gut [Internet]. 2009 [cited 2021 Jun 29];58:1538-1544. Available from:

https://pubmed.ncbi.nlm.nih.gov/19625277/

6. Marzuillo P, del Giudice EM, Santoro N. Pediatric fatty liver disease: Role of ethnicity and genetics. World Journal of Gastroenterology [Internet]. 2014 [cited 2021 Jun 29];20:7347-7355. Available from: /pmc/articles/PMC4064080/

7. Schwimmer JB, Ugalde-Nicalo P, Welsh JA, Angeles JE, Cordero M, Harlow KE, et al. Effect of a Low Free Sugar Diet vs Usual Diet on Nonalcoholic Fatty Liver Disease in Adolescent Boys: A Randomized Clinical Trial. JAMA - Journal of the American Medical Association [Internet]. 2019 [cited 2021 Jun 29];321:256-265. Available from: https://pubmed.ncbi.nlm.nih.gov/30667502/

8. van Name MA, Savoye M, Chick JM, Galuppo BT, Feldstein AE, Pierpont B, et al. A low $\omega-6$ to $\omega-3$ PUFA ratio (n-6:n-3 PUFA) diet to treat fatty liver disease in obese youth. Journal of Nutrition [Internet]. 2020 [cited 2021 Jun 29];159:2314-2321. Available from: /pmc/articles/PMC7467848/

9. Hrncir T, Hrncirova L, Kverka M, Hromadka R, Machova V, Trckova E, et al. Gut Microbiota and NAFLD: Pathogenetic Mechanisms, Microbiota Signatures, and Therapeutic Interventions. Microorganisms [Internet]. 2021 [cited 2021 Jun 29];9:957. Available from: /pmc/articles/PMC8146698/

10. Sharpton SR, Schnabl B, Knight R, Loomba R. Current Concepts, Opportunities, and Challenges of Gut Microbiome-Based Personalized Medicine in Nonalcoholic Fatty Liver Disease [Internet]. Cell Metabolism. 2021 [cited 2021 Jun 29];33:21-32. Available from: https://pubmed.ncbi.nlm.nih.gov/33296678/

11. Loomba R, Seguritan V, Li W, Long T, Klitgord N, Bhatt A, et al. Gut Microbiome-Based Metagenomic Signature for Non-invasive Detection of Advanced Fibrosis in Human Nonalcoholic Fatty Liver Disease. Cell Metabolism [Internet]. 2017 [cited 2021 Jun 29];25:1054-1062.e5. Available from: https://pubmed.ncbi.nlm.nih.gov/28467925/

12. Caussy C, Tripathi A, Humphrey G, Bassirian S, Singh S, Faulkner C, et al. A gut microbiome signature for cirrhosis due to nonalcoholic fatty liver disease. Nature Communications [Internet]. 2019 [cited 2021 Jun 29];10:1-9. Available from: https://doi.org/10.1038/s41467-019-09455-9

13. Kravetz AM, Testerman T, Galuppo B, Graf J, Pierpont B, Siebel S, et al. Effect of gut microbiota and PNPLA3 rs738409 variant on nonalcoholic fatty liver disease (NAFLD) in obese youth. Journal of Clinical Endocrinology and Metabolism [Internet]. 2020 [cited 2021 Jun 29];105. Available from: https://pubmed.ncbi.nlm.nih.gov/32561908/

14. Oh TG, Kim SM, Caussy C, Fu T, Guo J, Bassirian S, et al. A Universal Gut-Microbiome-Derived Signature Predicts Cirrhosis. Cell Metabolism. 2020;32:878-888.e6. 
15. Sharpton SR, Yong GJM, Terrault NA, Lynch S v. Gut Microbial Metabolism and Nonalcoholic Fatty Liver Disease. Hepatology Communications [Internet]. 2019 [cited 2021 Sep 21];3:29-43. Available from: https://onlinelibrary.wiley.com/doi/full/10.1002/hep4.1284

16. Anderson MJ. Permutational Multivariate Analysis of Variance (PERMANOVA). Wiley StatsRef: Statistics Reference Online [Internet]. 2017 [cited 2021 Jul 12];1-15. Available from: https://onlinelibrary.wiley.com/doi/full/10.1002/9781118445112.stat07841

17. Gloor GB, Reid G. Compositional analysis: a valid approach to analyze microbiome high-throughput sequencing data. https://doi.org/10.1139/cjm2015-0821 [Internet]. 2016 [cited 2021 Sep 23];62:692-703. Available from: https://cdnsciencepub.com/doi/abs/10.1139/cjm-2015-0821

18. Chierico F del, Nobili V, Vernocchi P, Russo A, Stefanis C de, Gnani D, et al. Gut microbiota profiling of pediatric nonalcoholic fatty liver disease and obese patients unveiled by an integrated meta-omics-based approach. Hepatology [Internet]. 2017 [cited 2021 Oct 6];65:451-464. Available from: https://onlinelibrary.wiley.com/doi/full/10.1002/hep.28572

19. Gilbert J, Blaser MJ, Caporaso JG, Jansson J, Lynch S v., Knight R. Current understanding of the human microbiome. Nature medicine [Internet]. 2018 [cited 2021 Aug 25];24:392. Available from: /pmc/articles/PMC7043356/

20. Senghor B, Sokhna C, Ruimy R, Lagier JC. Gut microbiota diversity according to dietary habits and geographical provenance. Human Microbiome Journal. 2018;7-8:1-9.

21. Sharpton S, Ajmera V, Loomba R. Emerging Role of the Gut Microbiome in Nonalcoholic Fatty Liver Disease: From Composition to Function. Clinical gastroenterology and hepatology: the official clinical practice journal of the American Gastroenterological Association [Internet]. 2019 [cited 2021 Sep 1];17:296. Available from: /pmc/articles/PMC6314895/

22. Lau E, Carvalho D, Freitas P. Gut Microbiota: Association with NAFLD and Metabolic Disturbances. BioMed Research International [Internet]. 2015 [cited 2021 Aug 29];2015. Available from: /pmc/articles/PMC4452311/

23. Liu R, Hong J, Xu X, Feng Q, Zhang D, Gu Y, et al. Gut microbiome and serum metabolome alterations in obesity and after weight-loss intervention. Nature Medicine 2017 23:7 [Internet]. 2017 [cited 2021 Aug 30];23:859-868. Available from: https://www.nature.com/articles/nm.4358

24. Hiippala K, Barreto G, Burrello C, Diaz-Basabe A, Suutarinen M, Kainulainen V, et al. Novel Odoribacter splanchnicus Strain and Its Outer Membrane Vesicles Exert Immunoregulatory Effects in vitro. Frontiers in Microbiology. 2020;0:2906.

25. Price CE, O'toole GA. The Gut-Lung Axis in Cystic Fibrosis. 2021 [cited 2021 Sep 22];Available from: https://doi.org/10.1128/JB.00311-21.

26. Jeffery IB, O'toole PW, Hman LO“, Claesson MJ, Deane J, Quigley EMM, et al. An irritable bowel syndrome subtype defined by species-specific alterations in faecal microbiota. [cited 2021 Sep 22];Available from: http://gut.bmj.

27. T T, T K, H T, K N. Fusicatenibacter saccharivorans gen. nov., sp. nov., isolated from human faeces. International journal of systematic and evolutionary microbiology [Internet]. 2013 [cited 2021 Oct 10];63:3691-3696. Available from: https://pubmed.ncbi.nlm.nih.gov/23625266/

28. Matsuyama M, Morrison M, Cao K-AL, Pruilh S, Davies PSW, Wall C, et al. Dietary intake influences gut microbiota development of healthy Australian children from the age of one to two years. Scientific Reports 2019 9:1 [Internet]. 2019 [cited 2021 Oct 10];9:1-11. Available from: https://www.nature.com/articles/s41598-019-48658-4

29. JGI IMG Actinomyces sp. ICM47 [Internet]. 2013 [cited 2021 Oct 11];Available from: https://img.jgi.doe.gov/cgi-bin/m/main.cgi? section=TaxonDetail\&page=taxonDetail\&taxon_oid=2537561777

30. Yamakado M, Tanaka T, Nagao K, Imaizumi A, Komatsu M, Daimon T, et al. Plasma amino acid profile associated with fatty liver disease and cooccurrence of metabolic risk factors. Scientific Reports 2017 7:1 [Internet]. 2017 [cited 2021 Aug 30];7:1-9. Available from:

https://www.nature.com/articles/s41598-017-14974-w

31. Goffredo M, Santoro N, Tricò D, Giannini C, D’Adamo E, Zhao H, et al. A Branched-Chain Amino Acid-Related Metabolic Signature Characterizes Obese Adolescents with Non-Alcoholic Fatty Liver Disease. Nutrients [Internet]. 2017 [cited 2021 Aug 30];9. Available from: /pmc/articles/PMC5537762/

32. Vinolo MAR, Rodrigues HG, Nachbar RT, Curi R. Regulation of Inflammation by Short Chain Fatty Acids. Nutrients [Internet]. 2011 [cited 2021 Sep 23];3:858. Available from: /pmc/articles/PMC3257741/

33. Parada Venegas D, de la Fuente MK, Landskron G, González MJ, Quera R, Dijkstra G, et al. Short Chain Fatty Acids (SCFAs)-Mediated Gut Epithelial and Immune Regulation and Its Relevance for Inflammatory Bowel Diseases. Frontiers in Immunology. 2019;0:277.

34. Derrien M, Alvarez A-S, Vos WM de. The Gut Microbiota in the First Decade of Life. Trends in Microbiology [Internet]. 2019 [cited 2021 Sep 1];27:9971010. Available from: http://www.cell.com/article/S0966842X19302148/fulltext

35. Gupta VK, Paul S, Dutta C. Geography, Ethnicity or Subsistence-Specific Variations in Human Microbiome Composition and Diversity. Frontiers in Microbiology. 2017;0:1162. 
36. Ranjan R, Rani A, Metwally A, McGee HS, Perkins DL. Analysis of the microbiome: Advantages of whole genome shotgun versus $16 \mathrm{~S}$ amplicon sequencing. Biochemical and biophysical research communications [Internet]. 2016 [cited 2021 Sep 1];469:967. Available from: /pmc/articles/PMC4830092/

37. Brumfield KD, Huq A, Colwell RR, Olds JL, Leddy MB. Microbial resolution of whole genome shotgun and $16 \mathrm{~S}$ amplicon metagenomic sequencing using publicly available NEON data. PLOS ONE [Internet]. 2020 [cited 2021 Sep 1];15:e0228899. Available from: https://journals.plos.org/plosone/article? $\mathrm{id}=10.1371$ /journal.pone. 0228899

38. Peterson D, Bonham KS, Rowland S, Pattanayak CW, Consortium R, Klepac-Ceraj V, et al. Comparative Analysis of 16S rRNA Gene and Metagenome Sequencing in Pediatric Gut Microbiomes. Frontiers in Microbiology. 2021;0:1651.

39. Durazzi F, Sala C, Castellani G, Manfreda G, Remondini D, de Cesare A. Comparison between 16S rRNA and shotgun sequencing data for the taxonomic characterization of the gut microbiota. Scientific Reports 2021 11:1 [Internet]. 2021 [cited 2021 Sep 1];11:1-10. Available from:

https://www.nature.com/articles/s41598-021-82726-y

40. Tricò D, Caprio S, Umano GR, Pierpont B, Nouws J, Galderisi A, et al. Metabolic Features of Nonalcoholic Fatty Liver (NAFL) in Obese Adolescents: Findings From a Multiethnic Cohort. Hepatology (Baltimore, Md.) [Internet]. 2018 [cited 2021 Aug 8];68:1376. Available from: /pmc/articles/PMC6173637/

41. Galderisi A, Giannini C, Weiss R, Kim G, Shabanova V, Santoro N, et al. Trajectories of changes in glucose tolerance in a multiethnic cohort of obese youths: An observational prospective analysis. The Lancet. Child \& adolescent health [Internet]. 2018 [cited 2021 Aug 8];2:726. Available from: /pmc/articles/PMC6190831/

42. Hershkop K, Besor O, Santoro N, Pierpont B, Caprio S, Weiss R. Adipose Insulin Resistance in Obese Adolescents Across the Spectrum of Glucose Tolerance. The Journal of Clinical Endocrinology and Metabolism [Internet]. 2016 [cited 2021 Oct 24];101:2423. Available from: /pmc/articles/PMC4891802/

43. Beghini F, Mclver LJ, Blanco-Míguez A, Dubois L, Asnicar F, Maharjan S, et al. Integrating taxonomic, functional, and strain-level profiling of diverse microbial communities with bioBakery 3. eLife. 2021;10.

44. R Core Team. R: A language and environment for statistical computing. 2017;

45. McMurdie PJ, Holmes S. phyloseq: An R Package for Reproducible Interactive Analysis and Graphics of Microbiome Census Data. PLoS ONE [Internet]. 2013 [cited 2019 Nov 15];8:e61217. Available from: https://dx.plos.org/10.1371/journal.pone.0061217

46. Barnett D. david-barnett/microViz: microViz 0.7.7. 2021 [cited 2021 Jun 18];Available from: https://zenodo.org/record/4898887

47. Mandal S, van Treuren W, White RA, Eggesb $\varnothing$ M, Knight R, Peddada SD. Analysis of composition of microbiomes: a novel method for studying microbial composition. Microbial Ecology in Health \& Disease [Internet]. 2015 [cited 2021 Jun 18];26. Available from: https://pubmed.ncbi.nlm.nih.gov/26028277/

48. Kaul A, Mandal S, Davidov O, Peddada SD. Analysis of microbiome data in the presence of excess zeros. Frontiers in Microbiology [Internet]. 2017 [cited 2021 Jun 18];8:2114. Available from: www.frontiersin.org

49. Lin H, Peddada S das. Analysis of compositions of microbiomes with bias correction. Nature Communications [Internet]. 2020 [cited 2021 Jun 18];11:1-11. Available from: https://doi.org/10.1038/s41467-020-17041-7

50. microbiome/microbiome: microbiome R package [Internet]. [cited 2021 Jun 18];Available from: https://github.com/microbiome/microbiome

51. Franzosa EA, Mclver LJ, Rahnavard G, Thompson LR, Schirmer M, Weingart G, et al. Species-level functional profiling of metagenomes and metatranscriptomes. Nature Methods [Internet]. 2018 [cited 2021 Jun 18];15:962-968. Available from: https://doi.org/10.1038/s41592-018-0176-y

52. Suzek BE, Huang H, McGarvey P, Mazumder R, Wu CH. UniRef: Comprehensive and non-redundant UniProt reference clusters. Bioinformatics [Internet]. 2007 [cited 2021 Jun 18];23:1282-1288. Available from: http://www.uniprot.org,aswellasfordownloadatftp://ftp.uniprot.org/pub/databases/uniprot/uniref

53. Suzek BE, Wang Y, Huang H, McGarvey PB, Wu CH. UniRef clusters: A comprehensive and scalable alternative for improving sequence similarity searches. Bioinformatics [Internet]. 2015 [cited 2021 Jun 18];31:926-932. Available from:

http://www.uniprot.org/unirefandftp://ftp.uniprot.org/pub/databases/uniprot/uniref.BLASTsearchesagainstUniRefareavailableathttp://www.uniprot.org/blast/

54. Mallick H, Rahnavard A, Mclver LJ, Ma S, Zhang Y, Tickle TL, et al. Multivariable Association Discovery in Population-scale Meta-omics Studies 3. bioRxiv [Internet]. 2021 [cited 2021 Jun 18];2021.01.20.427420. Available from: https://doi.org/10.1101/2021.01.20.427420

\section{Tables}

Table 1. Characteristics of study population by NAFLD status $(\mathrm{N}=36)$. 


\begin{tabular}{|c|c|c|c|}
\hline & \multicolumn{3}{|l|}{ NAFLD Status } \\
\hline & Negative $(n=18)$ & Positive (n=18) & $p$ \\
\hline \multicolumn{4}{|l|}{ Clinical Features } \\
\hline Age (years) ${ }^{1}$ & $12.22 \pm 3.02$ & $12.54 \pm 2.42$ & 0.7265 \\
\hline Gender (M/F) & $8 / 10(44.44 \% / 55.56 \%)$ & $11 / 7(61.11 \% / 38.89 \%)$ & 0.3166 \\
\hline \multirow[t]{2}{*}{ Race $^{3}$} & $6 / 4 / 6 / 1 / 1$ & $6 / 2 / 9 / 0 / 1$ & 0.6868 \\
\hline & $(33.33 \% / 22.22 \% / 33.33 \% / 5.56 \% / 5.56 \%)$ & $(33.33 \% / 11.11 \% / 50 \% / 0 \% / 5.56 \%)$ & \\
\hline Glucose Tolerance (NGT/IGT) 2,4 & $15 / 3$ & $10 / 5$ & 0.2660 \\
\hline $\operatorname{BMI}\left(\mathrm{kg} / \mathrm{m}^{2}\right)^{2,4}$ & $30.26 \pm 7.04$ & $35.21 \pm 6.82$ & 0.0386 \\
\hline BMI z-score ${ }^{2}$ & $2.09 \pm 0.48$ & $2.46 \pm 0.28$ & 0.0129 \\
\hline Body fat $(\%)^{2}$ & $41.34 \pm 10.23$ & $46.58 \pm 7.86$ & 0.1100 \\
\hline \multicolumn{4}{|l|}{ Glucose Metabolism } \\
\hline Fasting glucose (mg/dL) ${ }^{2}$ & $89.75 \pm 5.37$ & $94.53 \pm 8.31$ & 0.0549 \\
\hline Fasting insulin $(\mathrm{uU} / \mathrm{mL})^{2}$ & $21.69 \pm 5.58$ & $50.53 \pm 30.40$ & 0.0004 \\
\hline 2-h glucose $(\mathrm{mg} / \mathrm{dL})^{2}$ & $118.11 \pm 25.35$ & $130.80 \pm 33.50$ & 0.2249 \\
\hline Hemoglobin $\mathrm{A}_{1 \mathrm{C}}(\%)^{2}$ & $5.51 \pm 0.18$ & $5.56 \pm 0.30$ & 0.5491 \\
\hline WBISI ${ }^{2,4}$ & $2.45 \pm 0.61$ & $1.24 \pm 1.24$ & 0.0003 \\
\hline$|G|^{2,4}$ & $4.25 \pm 3.43$ & $6.42 \pm 4.38$ & 0.1281 \\
\hline $\mathrm{DI} 2,4$ & $8.24 \pm 4.47$ & $8.03 \pm 8.32$ & 0.9298 \\
\hline \multicolumn{4}{|l|}{ Lipid Profile } \\
\hline Total Cholesterol $(\mathrm{mg} / \mathrm{dL})^{2}$ & $143.72 \pm 28.39$ & $152.21 \pm 24.65$ & 0.3816 \\
\hline HDL Cholesterol $(\mathrm{mg} / \mathrm{dL})^{2,4}$ & $43.94 \pm 8.21$ & $44.29 \pm 10.41$ & 0.9180 \\
\hline LDL Cholesterol $(\mathrm{mg} / \mathrm{dL}){ }^{2,4}$ & $84.28 \pm 27.19$ & $85.64 \pm 19.65$ & 0.8754 \\
\hline Triglycerides (mg/dL) ${ }^{2}$ & $77.17 \pm 28.24$ & $111.14 \pm 68.63$ & 0.0658 \\
\hline \multicolumn{4}{|l|}{ Liver Function } \\
\hline Alanine Transaminase $(\mathrm{U} / \mathrm{L})^{2}$ & $18.33 \pm 7.27$ & $40.14 \pm 33.80$ & 0.0121 \\
\hline Aspartate Transaminase $(\mathrm{U} / \mathrm{L})^{2}$ & $20.83 \pm 4.99$ & $32.06 \pm 26.93$ & 0.0925 \\
\hline \multicolumn{4}{|l|}{ Body Fat Composition } \\
\hline Visceral (cm2) & $52.61 \pm 24.86$ & $77.24 \pm 24.03$ & 0.0047 \\
\hline Deep Subcutaneous (cm2) & $184.98 \pm 202.32$ & $171.37 \pm 58.03$ & 0.7855 \\
\hline Subcutaneous (cm2) ${ }^{2}$ & $467.91 \pm 215.92$ & $560.69 \pm 234.84$ & 0.2332 \\
\hline Superficial Subcutaneous (cm2) & $161.93 \pm 90.37$ & $155.98 \pm 96.29$ & 0.8323 \\
\hline Deep/Superficial Subcutaneous & $1.14 \pm 0.59$ & $1.20 \pm 0.30$ & 0.7209 \\
\hline Hepatic Fat Fraction (\%) & $1.23 \pm 1.74$ & $20.86 \pm 11.36$ & $<0.0001$ \\
\hline \multicolumn{4}{|l|}{${ }^{1}$ Mean \pm SD } \\
\hline \multicolumn{4}{|c|}{$23,1,3,3,3,3,3,5,3,4,4,4,4,4,4,4,1$ missing values } \\
\hline \multicolumn{4}{|c|}{${ }^{3}$ White or Caucasian/Black or African American/Hispanics/Others/Asian } \\
\hline \multicolumn{4}{|c|}{$\begin{array}{l}{ }^{4} \mathrm{NGT} \text { - Normal Glucose Tolerance, IGT - Impaired Glucose Tolerance, BMI - Body Mass Index, WBISI - Whole-body Insulin Sensitivity Index, IGI - } \\
\text { Insulinogenic Index, DI - Disposition Index, HDL Cholesterol - High-Density Lipoprotein Cholesterol, LDL Cholesterol - Low-Density Lipoprotein } \\
\text { Cholesterol }\end{array}$} \\
\hline
\end{tabular}


Table 2: ANCOM-BC Results.

\begin{tabular}{|c|c|c|c|c|c|}
\hline Species $^{1}$ & Coefficient (beta) & Standard ErTor (SE) & Test Statistic (W) & p-value & Adjusted p-value (q) \\
\hline Actinomyces sp. ICM47 & 4.42267 & 0.98863 & 4.47353 & 7.69375E-06 & $9.23250 \mathrm{E}-04$ \\
\hline Fusicatenibacter saccharivorans & 1.98506 & 0.55559 & 3.57288 & $3.53079 \mathrm{E}-04$ & 4.16633E-02 \\
\hline Romboutsia ilealis & 4.16014 & 1.17304 & 3.54645 & $3.90464 \mathrm{E}-04$ & $4.56842 \mathrm{E}-02$ \\
\hline Bacteroides thetaiotaomicron & -9.03164 & 1.88506 & -4.79116 & $1.65800 \mathrm{E}-06$ & $1.98000 \mathrm{E}-04$ \\
\hline \multicolumn{6}{|l|}{ Genus $^{1}$} \\
\hline Bacteroides & -3.02484 & 0.53084 & -5.69821 & $1.21073 \mathrm{E}-08$ & $8.47514 \mathrm{E}-07$ \\
\hline Flavonifractor & -3.20868 & 0.80108 & -4.00544 & 6.19029E-05 & 4.27130E-03 \\
\hline \multicolumn{6}{|l|}{ Family ${ }^{1}$} \\
\hline Bacteroidaceae & -2.85427 & 0.58112 & -4.91166 & $9.03083 \mathrm{E}-07$ & $3.07048 \mathrm{E}-05$ \\
\hline Odoribacteraceae & -5.52603 & 1.38351 & -3.99422 & $6.49073 \mathrm{E}-05$ & 2.14194E-03 \\
\hline \multicolumn{6}{|l|}{ Order $^{1}$} \\
\hline Bacteroidales & -2.67369 & 0.47544 & -5.62363 & 1.86985E-08 & $3.55271 \mathrm{E}-07$ \\
\hline \multicolumn{6}{|l|}{ Class $^{1}$} \\
\hline Bacteroidia & -2.78369 & 0.44122 & -6.30911 & $2.80639 \mathrm{E}-10$ & 3.92895E-09 \\
\hline Tissierellia & -3.77561 & 1.30288 & -2.89790 & 3.75667E-03 & 4.88367E-02 \\
\hline \multicolumn{6}{|l|}{ Phylum ${ }^{1}$} \\
\hline Bacteroidetes & -2.84922 & 0.41898 & -6.80041 & $1.04323 \mathrm{E}-11$ & $6.25940 \mathrm{E}-11$ \\
\hline
\end{tabular}

${ }^{1}$ Only taxa appearing in $25 \%$ of samples were included in each taxonomic level's analysis.

Table 3: Elevated Metabolic Pathways.

${ }^{1}$ Prevalence - the number of samples this particular pathway was detected in.

\section{Figures}




\begin{tabular}{|c|c|c|c|c|c|c|c|}
\hline Pathway & Class & $\begin{array}{l}\text { Group } \\
\text { Elevated }\end{array}$ & Coefficient & $\begin{array}{l}\text { Standard } \\
\text { Error }\end{array}$ & Prevalence $^{1}$ & $\mathbf{p}$ & q \\
\hline COA PWY: coenzyme A biosynthesis I: prokaryotic & Biosynthesis & $\begin{array}{l}\text { NAFLD- } \\
\text { positive }\end{array}$ & 0.257 & 0.052 & 36 & 0.00002 & 0.01318 \\
\hline $\begin{array}{l}\text { GLYCOGENSYNTH PWY: glycogen biosynthesis I: } \\
\text { from ADP D Glucose }\end{array}$ & Biosynthesis & $\begin{array}{l}\text { NAFLD- } \\
\text { positive }\end{array}$ & 0.751 & 0.142 & 36 & 0.00001 & 0.01318 \\
\hline $\begin{array}{l}\text { PWY 4242: pantothenate and coenzyme A } \\
\text { biosynthesis III }\end{array}$ & Biosynthesis & $\begin{array}{l}\text { NAFLD- } \\
\text { positive }\end{array}$ & 0.285 & 0.056 & 36 & 0.00001 & 0.01318 \\
\hline $\begin{array}{l}\text { PWY 5100: pyruvate fermentation to acetate and } \\
\text { lactate II }\end{array}$ & Degradation/Utilization & $\begin{array}{l}\text { NAFLD- } \\
\text { positive }\end{array}$ & 0.747 & 0.149 & 36 & 0.00002 & 0.01318 \\
\hline $\begin{array}{l}\text { PWY 6471: peptidoglycan biosynthesis IV: } \\
\text { Enterococcus faecium }\end{array}$ & Biosynthesis & $\begin{array}{l}\text { NAFLD- } \\
\text { positive }\end{array}$ & 1.265 & 0.249 & 36 & 0.00001 & 0.01318 \\
\hline ARO PWY: chorismate biosynthesis I & Biosynthesis & $\begin{array}{l}\text { NAFLD- } \\
\text { positive }\end{array}$ & 0.304 & 0.068 & 36 & 0.00009 & 0.01343 \\
\hline $\begin{array}{l}\text { BRANCHED CHAIN AA SYN PWY: superpathway } \\
\text { of branched chain amino acid biosynthesis }\end{array}$ & Biosynthesis & $\begin{array}{l}\text { NAFLD- } \\
\text { positive }\end{array}$ & 0.268 & 0.063 & 36 & 0.00017 & 0.01343 \\
\hline $\begin{array}{l}\text { COMPLETE ARO PWY: superpathway of aromatic } \\
\text { amino acid biosynthesis }\end{array}$ & Biosynthesis & $\begin{array}{l}\text { NAFLD- } \\
\text { positive }\end{array}$ & 0.309 & 0.070 & 36 & 0.00009 & 0.01343 \\
\hline HSERMETANA PWY: L methionine biosynthesis III & Biosynthesis & $\begin{array}{l}\text { NAFLD- } \\
\text { positive }\end{array}$ & 0.472 & 0.099 & 36 & 0.00003 & 0.01343 \\
\hline $\begin{array}{l}\text { LACTOSECAT PWY: lactose and galactose } \\
\text { degradation I }\end{array}$ & Degradation/Utilization & $\begin{array}{l}\text { NAFLD- } \\
\text { positive }\end{array}$ & 1.592 & 0.379 & 36 & 0.00018 & 0.01343 \\
\hline PWY 5097: L lysine biosynthesis VI & Biosynthesis & $\begin{array}{l}\text { NAFLD- } \\
\text { positive }\end{array}$ & 0.216 & 0.051 & 36 & 0.00017 & 0.01343 \\
\hline PWY 5103: L isoleucine biosynthesis III & Biosynthesis & $\begin{array}{l}\text { NAFLD- } \\
\text { positive }\end{array}$ & 0.311 & 0.070 & 36 & 0.00009 & 0.01343 \\
\hline PWY 6270: isoprene biosynthesis I & Biosynthesis & $\begin{array}{l}\text { NAFLD- } \\
\text { positive }\end{array}$ & 0.562 & 0.121 & 36 & 0.00005 & 0.01343 \\
\hline $\begin{array}{l}\text { PWY 7221: guanosine ribonucleotides de novo } \\
\text { biosynthesis }\end{array}$ & Biosynthesis & $\begin{array}{l}\text { NAFLD- } \\
\text { positive }\end{array}$ & 0.237 & 0.051 & 36 & 0.00005 & 0.01343 \\
\hline $\begin{array}{l}\text { PWY 724: superpathway of L lysine: } L \text { threonine } \\
\text { and L methionine biosynthesis II }\end{array}$ & Biosynthesis & $\begin{array}{l}\text { NAFLD- } \\
\text { positive }\end{array}$ & 0.262 & 0.058 & 36 & 0.00006 & 0.01343 \\
\hline $\begin{array}{l}\text { PWY 7357: thiamine phosphate formation from } \\
\text { pyrithiamine and oxythiamine: yeast }\end{array}$ & Biosynthesis & $\begin{array}{l}\text { NAFLD- } \\
\text { positive }\end{array}$ & 0.419 & 0.093 & 36 & 0.00008 & 0.01343 \\
\hline PWY 7560: methylerythritol phosphate pathway II & Biosynthesis & $\begin{array}{l}\text { NAFLD- } \\
\text { positive }\end{array}$ & 0.597 & 0.129 & 36 & 0.00005 & 0.01343 \\
\hline GLUTORN PWY: L ornithine biosynthesis I & Biosynthesis & $\begin{array}{l}\text { NAFLD- } \\
\text { positive }\end{array}$ & 0.369 & 0.091 & 36 & 0.00028 & 0.01710 \\
\hline $\begin{array}{l}\text { METH ACETATE PWY: methanogenesis from } \\
\text { acetate }\end{array}$ & Energy Generation & $\begin{array}{l}\text { NAFLD- } \\
\text { positive }\end{array}$ & 1.690 & 0.421 & 36 & 0.00031 & 0.01780 \\
\hline $\begin{array}{l}\text { NONOXIPENT PWY: pentose phosphate pathway: } \\
\text { non oxidative branch: I }\end{array}$ & Energy Generation & $\begin{array}{l}\text { NAFLD- } \\
\text { positive }\end{array}$ & 0.606 & 0.154 & 36 & 0.00038 & 0.02008 \\
\hline PWY 6527: stachyose degradation & Degradation/Utilization & $\begin{array}{l}\text { NAFLD- } \\
\text { positive }\end{array}$ & 0.677 & 0.176 & 36 & 0.00051 & 0.02400 \\
\hline $\begin{array}{l}\text { P4 PWY: superpathway of L lysine: } L \text { threonine } \\
\text { and L methionine biosynthesis I }\end{array}$ & Biosynthesis & $\begin{array}{l}\text { NAFLD- } \\
\text { positive }\end{array}$ & 1.269 & 0.331 & 36 & 0.00051 & 0.02401 \\
\hline CALVIN PWY: Calvin Benson Bassham cycle & Biosynthesis & $\begin{array}{l}\text { NAFLD- } \\
\text { positive }\end{array}$ & 0.358 & 0.094 & 36 & 0.00057 & 0.02470 \\
\hline $\begin{array}{l}\text { PWY 5188: tetrapyrrole biosynthesis I: from } \\
\text { glutamate }\end{array}$ & Biosynthesis & $\begin{array}{l}\text { NAFLD- } \\
\text { positive }\end{array}$ & 0.792 & 0.209 & 36 & 0.00058 & 0.02470 \\
\hline $\begin{array}{l}\text { PWY 6121: } 5 \text { aminoimidazole ribonucleotide } \\
\text { biosynthesis I }\end{array}$ & Biosynthesis & $\begin{array}{l}\text { NAFLD- } \\
\text { positive }\end{array}$ & 0.223 & 0.059 & 36 & 0.00058 & 0.02470 \\
\hline DAPLYSINESYN PWY: L lysine biosynthesis I & Biosynthesis & $\begin{array}{l}\text { NAFLD- } \\
\text { positive }\end{array}$ & 1.041 & 0.283 & 36 & 0.00080 & 0.02734 \\
\hline $\begin{array}{l}\text { PWY 6163: chorismate biosynthesis from } 3 \\
\text { dehydroquinate }\end{array}$ & Biosynthesis & $\begin{array}{l}\text { NAFLD- } \\
\text { positive }\end{array}$ & 0.255 & 0.069 & 36 & 0.00078 & 0.02734 \\
\hline PWY 6606: guanosine nucleotides degradation II & Degradation/Utilization & $\begin{array}{l}\text { NAFLD- } \\
\text { positive }\end{array}$ & 0.869 & 0.236 & 36 & 0.00080 & 0.02734 \\
\hline SER GLYSYN PWY: superpathway of L serine and & Biosynthesis & NAFLD- & 0.439 & 0.118 & 36 & 0.00074 & 0.02734 \\
\hline
\end{tabular}

Page 12/15 


\begin{tabular}{|c|c|c|c|c|c|c|c|}
\hline \multicolumn{2}{|l|}{ glycine biosynthesis I } & \multicolumn{6}{|l|}{ positive } \\
\hline ARGININE SYN4 PWY: L ornithine biosynthesis II & Biosynthesis & $\begin{array}{l}\text { NAFLD- } \\
\text { negative }\end{array}$ & -3.235 & 0.636 & 32 & 0.00001 & 0.01318 \\
\hline HISDEG PWY: L histidine degradation I & Degradation/Utilization & $\begin{array}{l}\text { NAFLD- } \\
\text { negative }\end{array}$ & -1.876 & 0.383 & 36 & 0.00002 & 0.01318 \\
\hline $\begin{array}{l}\text { PWY 1269: CMP } 3 \text { deoxy D manno octulosonate } \\
\text { biosynthesis }\end{array}$ & Biosynthesis & $\begin{array}{l}\text { NAFLD- } \\
\text { negative }\end{array}$ & -1.837 & 0.373 & 36 & 0.00002 & 0.01318 \\
\hline PWY 5973: cis vaccenate biosynthesis & Biosynthesis & $\begin{array}{l}\text { NAFLD- } \\
\text { negative }\end{array}$ & -1.440 & 0.288 & 36 & 0.00002 & 0.01318 \\
\hline PWY 7663: gondoate biosynthesis: anaerobic & Biosynthesis & $\begin{array}{l}\text { NAFLD- } \\
\text { negative }\end{array}$ & -1.556 & 0.300 & 36 & 0.00001 & 0.01318 \\
\hline CITRULBIO PWY: L citrulline biosynthesis & Biosynthesis & $\begin{array}{l}\text { NAFLD- } \\
\text { negative }\end{array}$ & -2.367 & 0.540 & 35 & 0.00011 & 0.01343 \\
\hline $\begin{array}{l}\text { POLYISOPRENSYN PWY: polyisoprenoid } \\
\text { biosynthesis: E: coli }\end{array}$ & Biosynthesis & $\begin{array}{l}\text { NAFLD- } \\
\text { negative }\end{array}$ & -1.875 & 0.443 & 36 & 0.00017 & 0.01343 \\
\hline PWY 4984: urea cycle & Degradation/Utilization & $\begin{array}{l}\text { NAFLD- } \\
\text { negative }\end{array}$ & -2.402 & 0.547 & 35 & 0.00010 & 0.01343 \\
\hline PWY 5030: L histidine degradation III & Degradation/Utilization & $\begin{array}{l}\text { NAFLD- } \\
\text { negative }\end{array}$ & -1.743 & 0.381 & 36 & 0.00006 & 0.01343 \\
\hline $\begin{array}{l}\text { PWY 5505: L glutamate and L glutamine } \\
\text { biosynthesis }\end{array}$ & Biosynthesis & $\begin{array}{l}\text { NAFLD- } \\
\text { negative }\end{array}$ & -2.094 & 0.483 & 35 & 0.00012 & 0.01343 \\
\hline $\begin{array}{l}\text { PWY0 845: superpathway of pyridoxal 5: } \\
\text { phosphate biosynthesis and salvage }\end{array}$ & Biosynthesis & $\begin{array}{l}\text { NAFLD- } \\
\text { negative }\end{array}$ & -2.621 & 0.560 & 33 & 0.00004 & 0.01343 \\
\hline $\begin{array}{l}\text { PYRIDOXSYN PWY: pyridoxal 5: phosphate } \\
\text { biosynthesis I }\end{array}$ & Biosynthesis & $\begin{array}{l}\text { NAFLD- } \\
\text { negative }\end{array}$ & -2.892 & 0.602 & 33 & 0.00003 & 0.01343 \\
\hline PWY 6859: all trans farnesol biosynthesis & Biosynthesis & $\begin{array}{l}\text { NAFLD- } \\
\text { negative }\end{array}$ & -2.037 & 0.493 & 36 & 0.00022 & 0.01441 \\
\hline $\begin{array}{l}\text { PWY 7539: } 6 \text { hydroxymethyl dihydropterin } \\
\text { diphosphate biosynthesis III: Chlamydia }\end{array}$ & Biosynthesis & $\begin{array}{l}\text { NAFLD- } \\
\text { negative }\end{array}$ & -1.551 & 0.376 & 36 & 0.00023 & 0.01461 \\
\hline PWY 5104: L isoleucine biosynthesis IV & Biosynthesis & $\begin{array}{l}\text { NAFLD- } \\
\text { negative }\end{array}$ & -2.218 & 0.546 & 33 & 0.00027 & 0.01663 \\
\hline $\begin{array}{l}\text { PWY 6147: } 6 \text { hydroxymethyl dihydropterin } \\
\text { diphosphate biosynthesis I }\end{array}$ & Biosynthesis & $\begin{array}{l}\text { NAFLD- } \\
\text { negative }\end{array}$ & -1.529 & 0.380 & 36 & 0.00030 & 0.01751 \\
\hline $\begin{array}{l}\text { PWY 7332: superpathway of UDP N } \\
\text { acetylglucosamine derived O antigen building } \\
\text { blocks biosynthesis }\end{array}$ & Biosynthesis & $\begin{array}{l}\text { NAFLD- } \\
\text { negative }\end{array}$ & -2.241 & 0.557 & 17 & 0.00030 & 0.01751 \\
\hline PWY 7392: taxadiene biosynthesis: engineered & Biosynthesis & $\begin{array}{l}\text { NAFLD- } \\
\text { negative }\end{array}$ & -1.781 & 0.448 & 36 & 0.00035 & 0.01877 \\
\hline TCA: TCA cycle I: prokaryotic & Energy Generation & $\begin{array}{l}\text { NAFLD- } \\
\text { negative }\end{array}$ & -0.855 & 0.218 & 36 & 0.00040 & 0.02066 \\
\hline $\begin{array}{l}\text { NAD BIOSYNTHESIS II: NAD salvage pathway III: } \\
\text { to nicotinamide riboside }\end{array}$ & Biosynthesis & $\begin{array}{l}\text { NAFLD- } \\
\text { negative }\end{array}$ & -2.145 & 0.582 & 33 & 0.00079 & 0.02734 \\
\hline $\begin{array}{l}\text { PWY 7282: } 4 \text { amino } 2 \text { methyl } 5 \\
\text { diphosphomethylpyrimidine biosynthesis II }\end{array}$ & Biosynthesis & $\begin{array}{l}\text { NAFLD- } \\
\text { negative }\end{array}$ & -1.428 & 0.388 & 35 & 0.00080 & 0.02734 \\
\hline
\end{tabular}




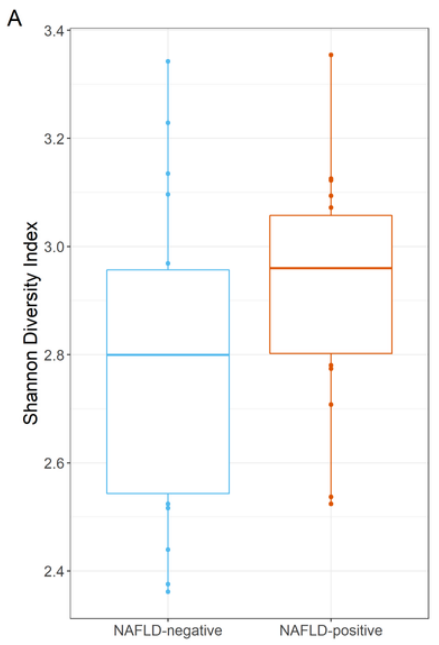

C

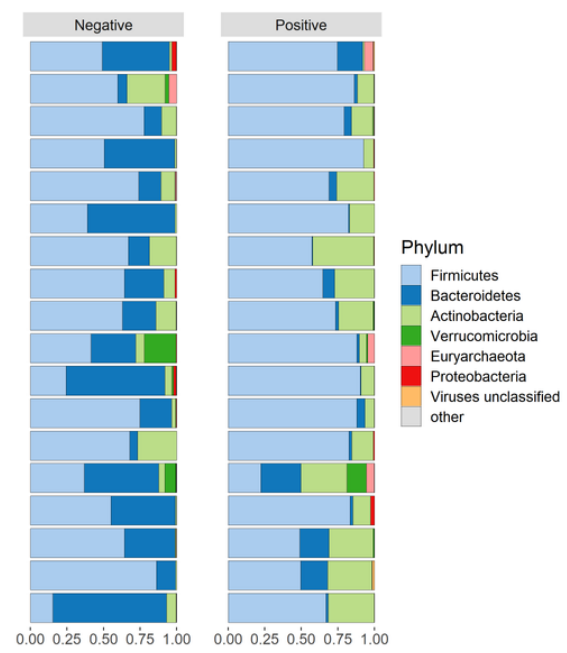

B

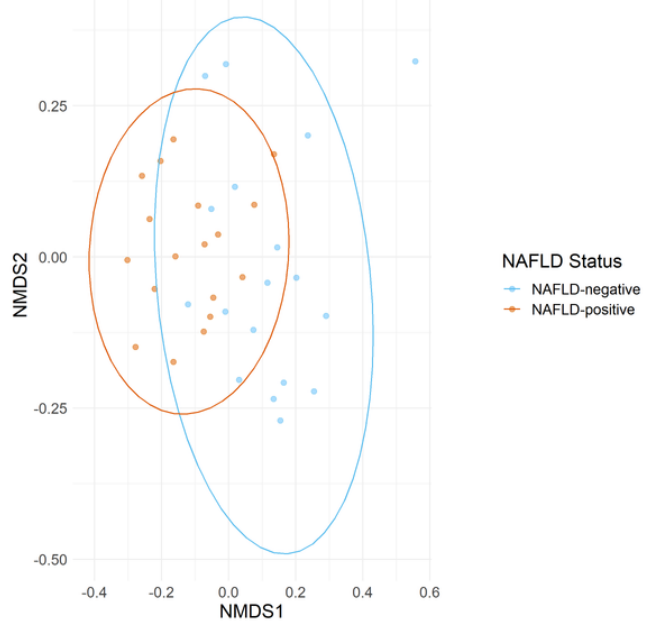

D

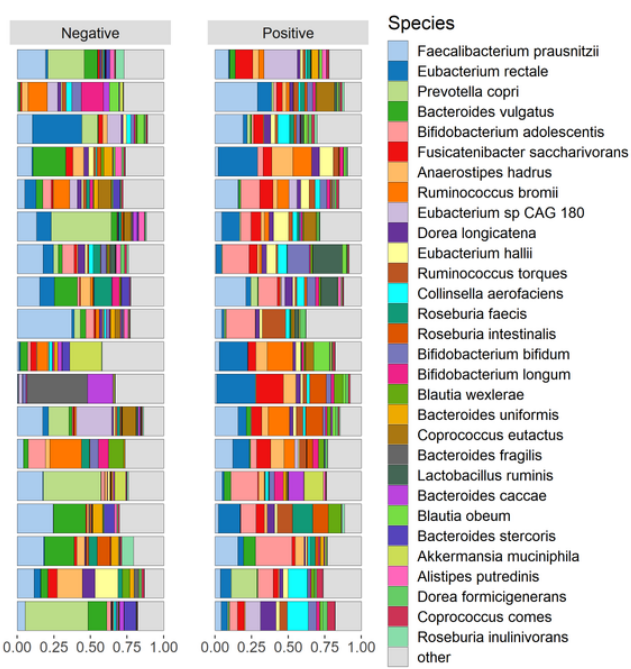

\section{Figure 1}

Microbiomes of 36 Obese Youth. (A) Shannon alpha diversity values are displayed as boxplots with median, interquartile range, and outliers marked. (B) BrayCurtis NMDS plot calculated at the species level using rarefied data. (C) Phylum-level and (D) species-level compositional bar plots are displayed with each horizontal bar representing an individual microbial community. NAFLD status is indicated above each column of bar plots. Other - all remaining taxa are grouped within this category. 
A

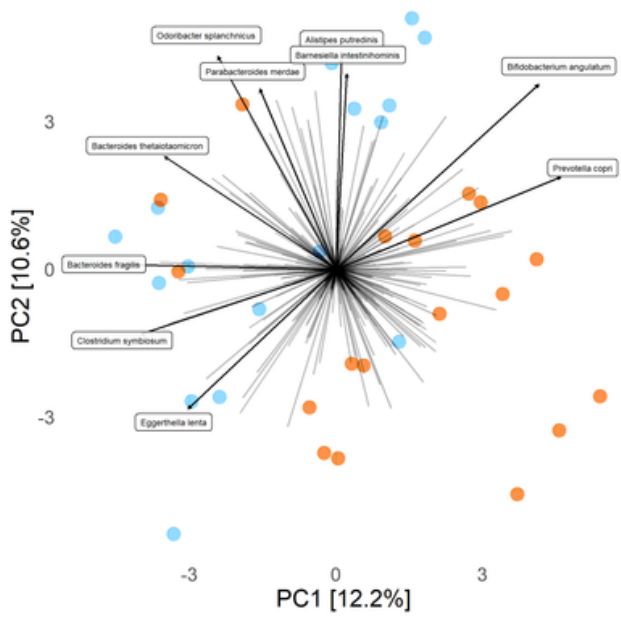

C

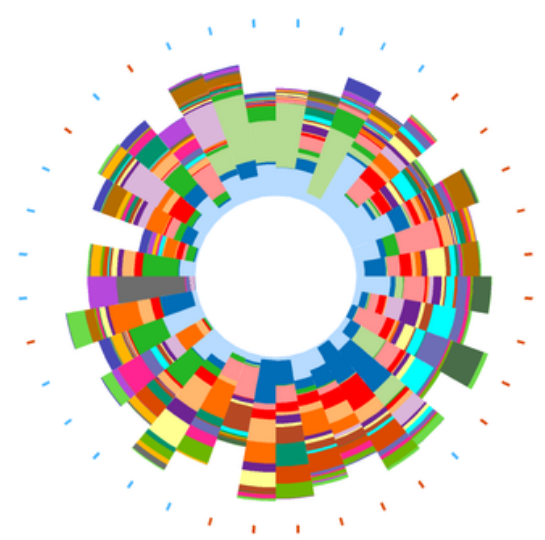

B

NAFLD Status

- NAFLD-negative

- NAFLD-positive

$$
\text { - Narl-mogotion }
$$

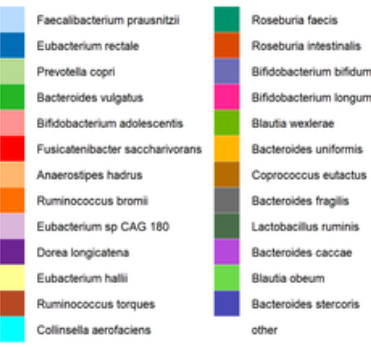

-

2

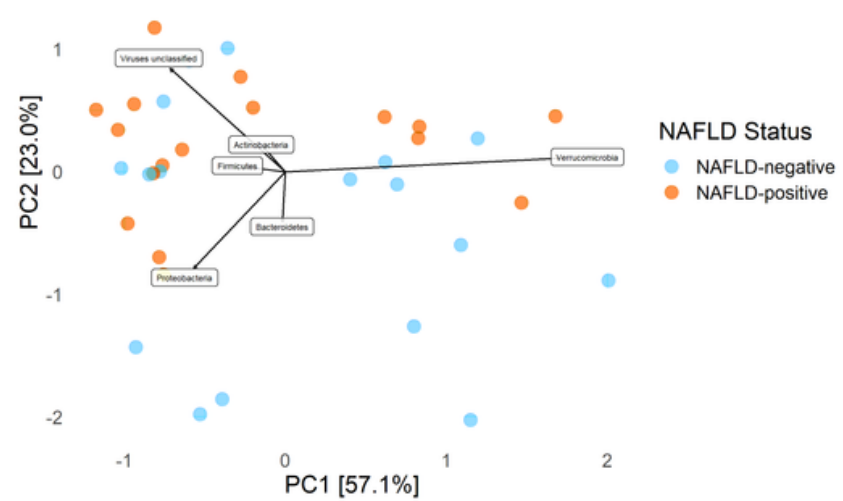

D

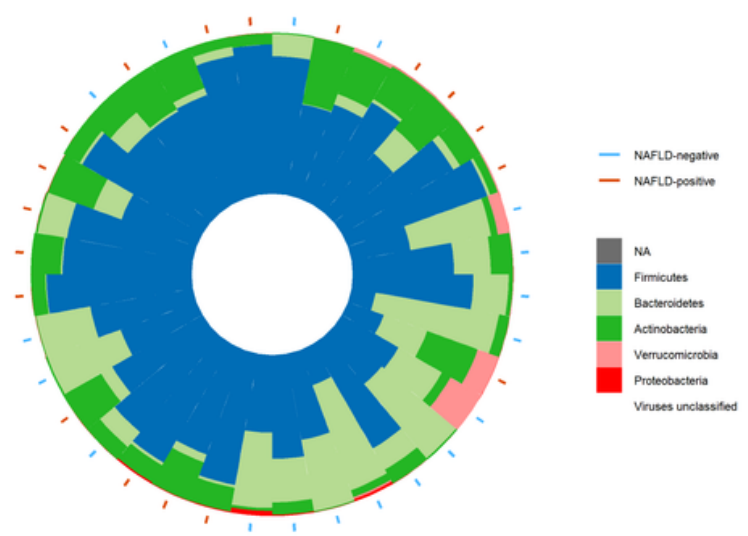

Figure 2

PCA Biplots with Taxa Arrows and Iris Plots. (A) Species-level PCA biplot with arrows indicating the abundance gradient for a particular species. The top 10 taxa variable contributors to the principal coordinates are shown. (B) Phylum-level PCA biplot with arrows indicating the abundance gradient for a particular phylum. The top 5 taxa variable contributors to the principal coordinates are shown. (C) Species-level iris plot showing the taxonomic composition of each sample with its position corresponding to its position in the associated biplot (above). The top 25 species are shown, and all remaining are grouped into the other category. (D) Phylum-level iris plot showing the taxonomic composition of each sample with its position corresponding to its position in the associated biplot (above). Orange circles on the periphery of the iris plots indicate NAFLD-positive individuals, blue circles indicate NAFLD- negative individuals. Genera had to appear in $25 \%$ of samples to be included.

\section{Supplementary Files}

This is a list of supplementary files associated with this preprint. Click to download.

- SupplementalMaterials.docx

- SupplementalTable1metabolicpathwayscolorcodedtaxa.xlsx 